\title{
Genetic and molecular mechanisms in multiple myeloma: a route to better understand disease pathogenesis and heterogeneity
}

This article was published in the following Dove Press journal:

The Application of Clinical Genetics

24 July 2010

Number of times this article has been viewed

\author{
Marie-Christine Kyrtsonis ${ }^{1,2}$ \\ Vassiliki Bartzis ${ }^{1,2}$ \\ Xenophon Papanikolaou' \\ Efstathios Koulieris ${ }^{1,2}$ \\ George Georgiou ${ }^{2}$ \\ Maria Dimou ${ }^{2}$ \\ Tatiana Tzenou ${ }^{1,2}$ \\ Panayiotis Panayiotidis ${ }^{2}$ \\ 'First Department of Propedeutic \\ Internal Medicine, ${ }^{2}$ Department \\ of Hematology, National and \\ Kapodistrian University of Athens, \\ Laikon Hospital, Greece
}

Correspondence: Marie-Christine Kyrtsonis

First Department of Propedeutic Internal Medicine, National and Kapodistrian University of Athens' School of Medicine, Laikon University Hospital, Agiou Thoma 17,

Athens II527, Greece

Tel +302107456261

Fax +302107788830

Email mck@ath.forthnet.gr

\begin{abstract}
Multiple myeloma (MM) is a heterogeneous plasma cell neoplasm presenting with a wide range of clinical manifestations. In spite of the availability of very performing treatment modalities, survival is highly varying, ranging from a few months to several years. Underlying genetic and microenvironmental mechanisms are thought to be responsible for clinical heterogeneity. Disease etiology is unknown but progresses in the understanding of its pathogenesis have shown that MM precursor cell transformation into a malignant one occurs in a multistep process. Possibly during class switch recombination a primary genetic event takes place. With the occurrence of additional events and the support of bone marrow microenvironmental cells, neoplastic plasma cells actively proliferate and disease behavior may change. Recurrent translocations involving the IgH locus (11q13, 4p16, 16q23, 21q12, and 6p21), deletions of chromosome 13, trisomies of chromosomes 3, 5, 9,11, 15, 19, and 21, and dysregulated expression of cyclin $\mathrm{D}$ genes, are considered initiating or primary events. Alterations related to further disease transformation and adverse prognosis are deletion of $17 \mathrm{p} 13, \mathrm{c}-m y c$ translocations, and gains of chromosome 1q21. In relation to the underlying genetic defects, disease subgroups are recognized. Accordingly treatment effectiveness may differ among groups. Intense research is ongoing in this field.
\end{abstract}

Keywords: myeloma, pathogenesis, genetic abnormalities, heterogeneity, prognosis

\section{Introduction}

Multiple myeloma (MM) is a hematological malignancy affecting terminally differentiated B-cells and characterized by bone marrow infiltration by plasma cells secreting a monoclonal compound ( $\operatorname{IgG}, \operatorname{IgA}, \operatorname{IgD}$, or light chains). The disease is heterogeneous with a wide range of clinical manifestations and differing outcomes driven by the genetic-biologic characteristics; survival consequently ranging from a few months to several years. Tremendous progress has been made in the understanding of MM pathogenesis over the past two decades. Biology-modulating approaches were made available and others are currently under development, giving hope for more efficient therapeutic strategies. However, complex disease mechanisms should still be decrypted or their interpretation, remaining controversial, is to be clarified.

Frequently, research on a particular item arises from observations and random findings, the value of which is subsequently established by analyzing their statistical impact in large series. In other instances, experiments are performed in disease-similar microsystems, ex vivo or in animal models, but may not exactly reflect the complex oncogenic processes that really take place and lead to veracity-like hypotheses. Accordingly, part of the enormous amount of knowledge acquired concerning MM genetics 
and molecular pathophysiology, should be further validated.

For the purposes of the present study, we will discuss the possible origin of the malignant plasma cell and the theoretical processes that sustain its proliferation, the heterogeneity of MM manifestations and its precursor condition monoclonal gammopathy of undetermined significance (MGUS) and the main genetic alterations that have been described to take place during disease progression as well as their presumed clinical impact.

\section{Hypotheses on the origin of the myeloma clone}

During B-cell developmental evolution, the rearrangement of immunoglobulin (Ig), heavy (IgH) and light chain ( $\mathrm{IgL})$ genes $\left(\mathrm{V}_{\mathrm{H}}-\mathrm{N}-\mathrm{D}_{\mathrm{H}}-\mathrm{J}_{\mathrm{H}}\right.$ on chromosome 14 and $\mathrm{V}_{\mathrm{L}}-\mathrm{N}-\mathrm{J}_{\mathrm{L}}$ on chromosomes 2 and 22 for $\kappa$ and $\lambda$ light chain respectively), take place. The process starts in the early pre-B-cell and leads to a unique $\operatorname{IgH}$ and $\operatorname{IgL}$ rearrangement. If the B-cell enters the germinal center (GC), it will undergo somatic hypermutation (SHM) and isotype class switch recombination (CSR), thus being selected to recognize a given antigen. Being a plasma cell, the MM cell precursor is considered a terminally differentiated B-lymphocyte that appears to have passed the normal steps, thus its immunoglobulin genes have undergone antigen selection, isotype switching, and SHM in a GC. The analysis of $\operatorname{IgH}$ gene sequences in MM showed extensive hypermutations without evidence of intraclonal variations confirming their GC provenance. ${ }^{1}$
The first genetic "event" of a multistep neoplastic process could take place at the time of CSR with the random occurrence of translocations to the $\mathrm{IgH}$ locus on chromosome $14 \mathrm{q} 32 .^{2}$ These translocations lead to the deregulation of an oncogene juxtaposed to them and result in immortalization of the clone. ${ }^{3}$ When leaving the GC, the precursor cell may either become an Ig-producing plasma cell or a memory B-lymphocyte. Studies on MM cell growth fraction have shown that the majority of neoplastic plasma cells are quiescent at least at diagnosis and that tumor growth is restricted to a specific cell subpopulation ${ }^{4}$ that is not fully documented, but could be memory cells. It was subsequently shown, using allele-specific oligonucleotide-based polymerase chain reactions that clonal cells expressing a B-cell rather that plasma cell phenotype are circulating in the blood and bone marrow (BM) of MM patients ${ }^{5}$ and it was suggested that these clonotypic cells were equivalent to memory B-cells with their normal self-renewal properties. This last hypothesis recently led to the concept of the MM stem cell. ${ }^{6}$ A possible genetic predisposition for the development of MM precursor cell is suggested by familial and population-based studies showing an increased risk, among relatives of MM and MGUS patients, to develop related malignancies. $^{7}$

The precursor memory clonotypic B-cell will selectively enter and bind to the BM milieu that actively contributes to its proliferation. A theoretical illustration of the evolution of the precursor cell to MM cell as well as its subsequent further evolution is shown in Figure 1.

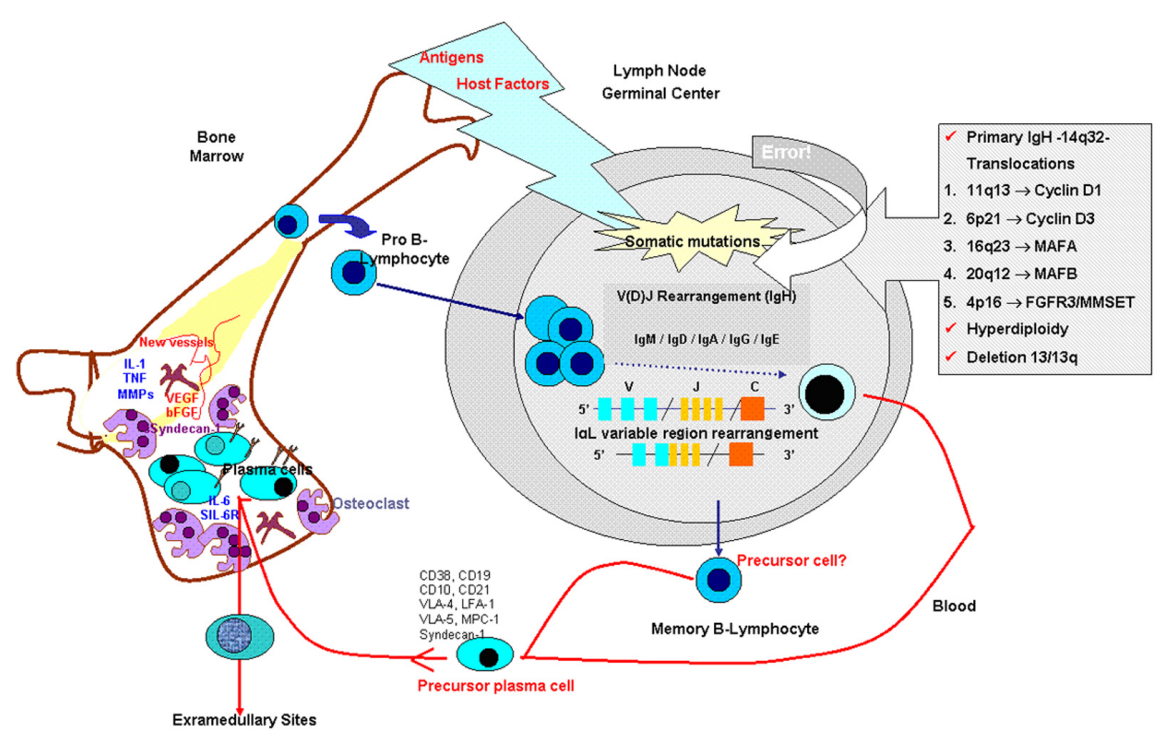

Figure I Hypothetic model of disease transformation. The MM precursor cell enters the GC and during CSR a primary genetic event occurs by error. The cell becomes a B-memory cell that carries its stigma and will further transform to an MGUS and ultimately MM cell with the occurrence of additional genetic events and the support of bone marrow microenvironmental cells. 


\section{The bone marrow microenvironment}

The initial almost exclusive homing in the BM can be explained on one hand by the presence of adhesion molecules on plasma cell surfaces (very late antigen-4 [VLA-4], leukocyte function-associated antigen-1, monocyte chemotatic protein-1, VLA-5, syndecan-1) and on the other by the ability of the BM microenvironment to promote malignant cells' proliferation. ${ }^{8,9}$ Triggered by plasma cells and stromal cells (SC) contact, the transcription factor, nuclear factor- $\mathrm{\kappa B}(\mathrm{NF}-\kappa \mathrm{B})$ activates and upregulates the expression of adhesion molecules such as intercellular adhesion molecule- 1 and vascular cell adhesion molecule-1 by both cell types, resulting in tighter binding. This stimulates, in turn, the production of a wide spectrum of cytokines that activate proliferative, antiapoptotic signalling pathways in MM cells and promote neo-angiogenesis and osteoclastogenesis. ${ }^{10,11}$ Within the BM milieu high levels of the complex interleukin- 6 and its soluble receptor (IL-6/ sIL-6R) constitute the main plasma cell growth factor during the initial steps of myeloma transformation. Later on, the malignant cell is transformed further and may become IL-6- and BM-independent.

As already mentioned two important processes in MM pathogenesis are angiogenesis and osteoclastogenesis. Neovascularization is needed to support the metabolic requirements of the neoplastic cells. The process includes among others, the production of angiogenic cytokines (vascular endothelial growth factor, basic fibroblast growth factor, hepatocyte growth factor, syndecan-1, angiopoietins, etc) ${ }^{12,13}$ that lead to the formation of new vessels. Bone destruction is mainly characterized by multiple osteolytic lesions caused by osteoclasts proliferating in bony areas adjacent to plasma cells; both cell types secrete soluble factors (osteoclast-activating factors including tumor necrosis factor- $\alpha[$ TNF- $\alpha]$, IL-1, IL-6, and macrophage inflammatory protein-1a) that sustain each other's activity. ${ }^{9}$ However, the main stimulator of osteoclast formation is a member of the TNF family, namely the receptor activator of NF- $\kappa$ B ligand (RANKL). ${ }^{14}$

It is possible that during intense BM-supported plasma cell proliferation, myeloma cells or a subset of them further transform rendering the disease more aggressive. In addition, it is unknown whether the BM milieu per se presents some kind of malignant transformation. Recent gene expression profiling studies suggest that gene expression of bone microenvironment cells (endothelial, mesenchymal) are different in MM patients and controls. ${ }^{15,16}$

\section{Monoclonal gammopathy of undetermined significance}

The prevailing hypothesis is that the clinical condition of MGUS reflects the occurrence of the initials genetic "events" of the multi-step neoplastic process involving the switch regions of the heavy chain locus ( $\operatorname{IgH})$ at $14 \mathrm{q} 32$; these translocations are considered a frequent primary finding because they are evenly found in individuals with MGUS and patients with MM. ${ }^{17}$ It should be mentioned here that hyperdiploidy, associated with multiple trisomies of specific chromosomes, has also been almost equally found in MGUS and MM and represent the base of another pathway of evolution with an unknown mechanism to date. ${ }^{18,19}$

Because translocations involving the IgH locus occur in numerous other B-cell lymphoproliferative disorders, MGUS may also evolve to other B-cell entities including indolent lymphomas and chronic lymphocytic leukemia. ${ }^{20,21}$

MGUS is characterized by the laboratory finding of a serum or urinary monoclonal component in the absence of signs, symptoms, end organ damage, and with a BM plasma cell infiltration of less than 10\%. By definition such a condition should be stable for at least six months. ${ }^{21,22}$ Patients are at increased risk of developing MM and related malignancies and the average risk of evolution is $1 \%$ per year. Furthermore, It was recently suggested that MGUS always precedes MM..$^{23,24}$ Serial measurements of serum-free light chains (FLC) and their ratio during follow-up examination of MGUS patients are useful for predicting upcoming disease evolution. ${ }^{23-25}$ This finding is possibly due to the increased sensitivity of the FLC assay to detect very low FLC amounts, ${ }^{26}$ but may also implicate the reflection of an unknown mechanism with a role in disease evolution and linked to the inappropriate FLC secretion by malignant plasma cells. Deletions of chromosome 13 are found in 70\% of myeloma patients with a documented pre-existing MGUS leading to the suspicion that this anomaly may constitute another crucial event of the multi-step process. ${ }^{27}$ It is possible that additional host factors, such as foreign and self antigens, ${ }^{28}$ contribute to the transformation process, perhaps by promoting BM reactive mechanisms leading to the secretion of cytokines and other factors that sustain plasma cell survival and growth.

\section{Multiple myeloma: heterogeneity of clinical manifestations, disease behavior, and survival}

Disease behavior in myeloma is highly variable. In many patients the disorder is indolent, symptom free and is 
called "smoldering or asymptomatic myeloma". In others it presents a more aggressive form with a variety of clinical symptoms, the so called "symptomatic myeloma"; in these cases the disease is frequently accompanied with significant morbidity. ${ }^{29}$

As plasma cells proliferate in the BM and their mass expands, osteoclasts' recruitment becomes active and bone resorption manifests by osseous pains, mainly back pain, followed in more advanced cases by compression or spontaneous fractures and even nerve paralysis. Moreover, the normal erythropoiesis is impaired, mostly by cytokines secreted upon plasma cells and stomal cells cross-talk, which leads to anemia, with patients complaining about fatigue, pallor, tachycardia and shortness of breath, and more rarely, thrombocytopenia with purpuric skin rash and leukocytopenia. The last, together with the malignant plasma cells producing their monoclonal paraprotein at the detriment of the normal plasma cell component leading to the decreased production of normal antibodies, cause immunosuppression, which is responsible for the recurrent infections that affect many patients. Another common presenting symptom is oliguria or anuria with swelling of extremities and vomiting due to renal insufficiency; these symptoms are mainly seen in light chain myeloma because the kidneys fail to metabolize or to reabsorb the increased FLC amounts excreted, so they may precipitate as casts and block the flow of urine..$^{30}$ The previ- ously mentioned bone destruction, along with renal failure, is responsible for hypercalcemia with symptoms ranging from constipation to even coma. Common MM presenting symptoms and their underlying mechanisms are shown in Figure 2. None of the above mentioned presenting symptoms is disease specific, neither will they present in all patients at the same time or sequence.

Symptomatic patients should be treated immediately before irreversible disease-related end-organ damage occurs. With conventional modalities the majority of patients enters some degree of response, but complete responses (CR) do not exceeded $5 \%$ while few patients are primary resistant, the median overall survival (OS) being three years. The addition of high-dose treatment (HDT) with autologous $\mathrm{BM}$ or stem cell rescue has increased the $\mathrm{CR}$ rate up to $30 \%-40 \%{ }^{31}$ and median OS to $4-5$ years. MM treatment armamentarium was considerably enriched over the past few years $^{32,33}$ with the approval of new drugs such as thalidomide, lenalidomide, and bortezomib that produce, alone and especially in combination with other agents, impressively high response rates, even in relapsed or resistant patients. ${ }^{34}$ However, even with these modalities, response duration is varying among patients and frequently unsatisfactory. ${ }^{35}$ Once again the heterogeneity in responses is partly due to the underlying biologic variations. Furthermore, genetic factors associated with resistance to specific treatments have

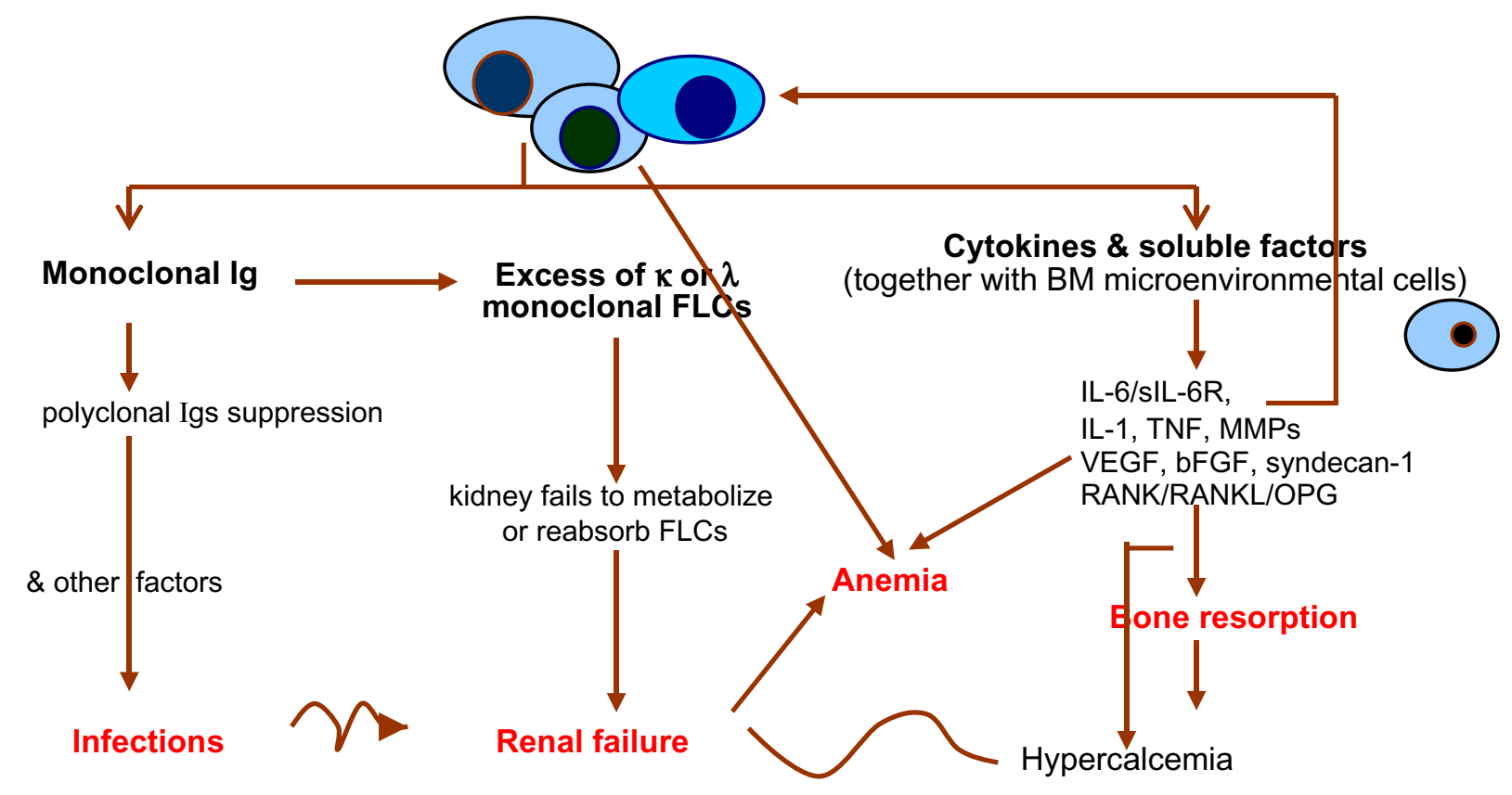

Figure 2 The malignant plasma cells, supported by the bone marrow microenvironment, are responsible for the clinical manifestations.

Abbreviations: bFGF, basic fibroblast growth factor; FLC, free light chains; IL, interleukin; TNF, tumor necrosis factor; VEGF, vascular endothelial growth factor; MMPs, matrix metalloproteineses; BM, bone marrow. 
been found. ${ }^{36}$ Ideally, treatment decisions should be guided by prognostic factors. ${ }^{37,38}$

\section{Genetic alterations}

In the BM the interactions between malignant plasma cells and their environment sustain plasma cell survival and proliferation. The oncogenic process continues given that $\mathrm{MM}$ cell is characterized by a profound genomic instability ${ }^{39}$ and subclones presenting additional genetic alterations appear. Chromosomal aberrations, numerical and structural, are a hallmark of MM and evolve over the course of the disease. ${ }^{40}$ Typically, previously treated and relapsed patients have a higher frequency of chromosome abnormalities compared with newly diagnosed ones. Although no single and pathognomonic genetic lesion has been identified for $\mathrm{MM}$, the presence of recurrent abnormalities with clinical and prognostic impact renders their contribution to disease pathobiology obvious.

All myelomas can be broadly divided into hyperdiploid and nonhyperdiploid. Patients with hyperdiploidy present with high chromosome number associated with trisomies of chromosomes $3,5,9,11,15,19$, and 21; they have a better overall survival. Hypodiploid myeloma is found in approximately $20 \%$ of the patients, pseudodiploid karyotypes in $15 \%$, and subtetradiploid in $5 \%$. Patients with nonhyperdiploid karyotypes present a high frequency of reciprocal translocations involving the $\operatorname{IgH}$ locus. ${ }^{41}$ These abnormalities deregulate juxtaposed genes; thus recurrent changes in the expression of a subset of genes may be partly responsible for disease heterogeneity, producing myeloma-specific unique clinical patterns. The main genetic findings recurrently found and that present clinical repercussions in MM are listed below and in Table 1. Recurrent trisomies or other findings with unknown implications are not discussed in the present context. It is interesting to observe that the abnormalities observed are not myeloma specific and may occur in B-cell lymphoproliferative disorders or even other neoplasias in which they may confer another phenotype than in MM, suggesting a role for additional factors.

\section{Technique mostly used for the detection of genetic abnormalities}

Chromosomal abnormalities are evaluated by conventional karyotype, the analysis of metaphasis of dividing cells. This technique cannot detect aberrations in most cases because myeloma cells are characterized by low proliferation rates (Figure 3).

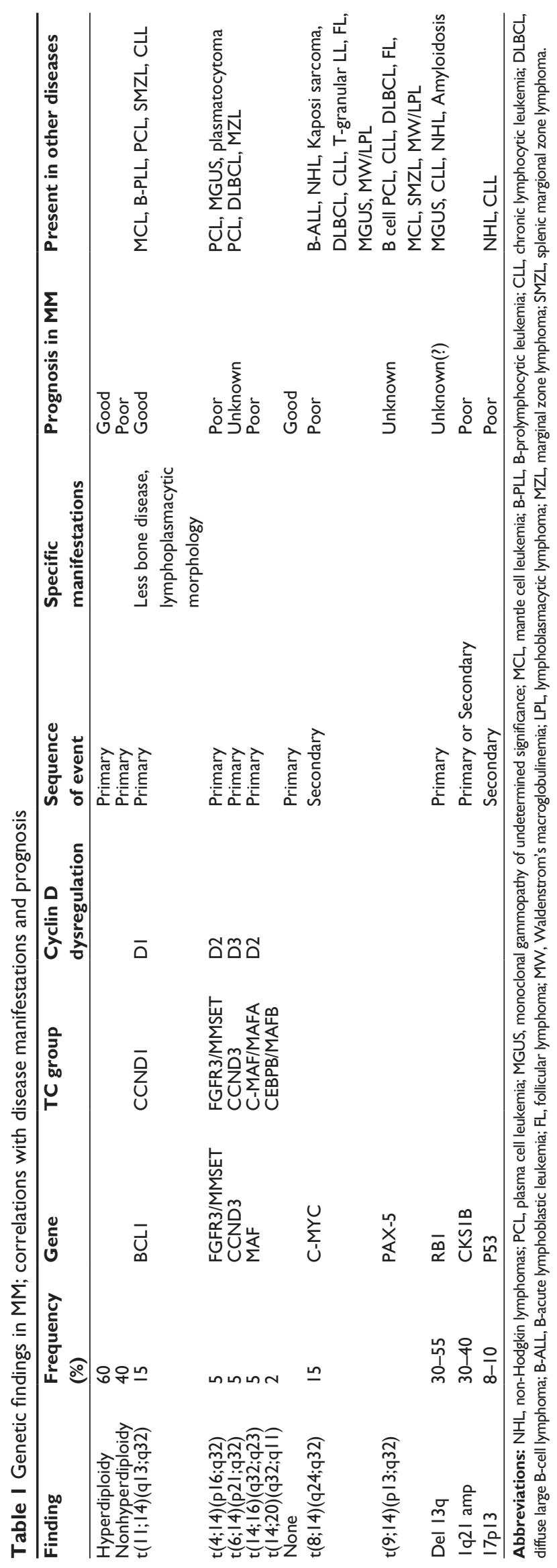




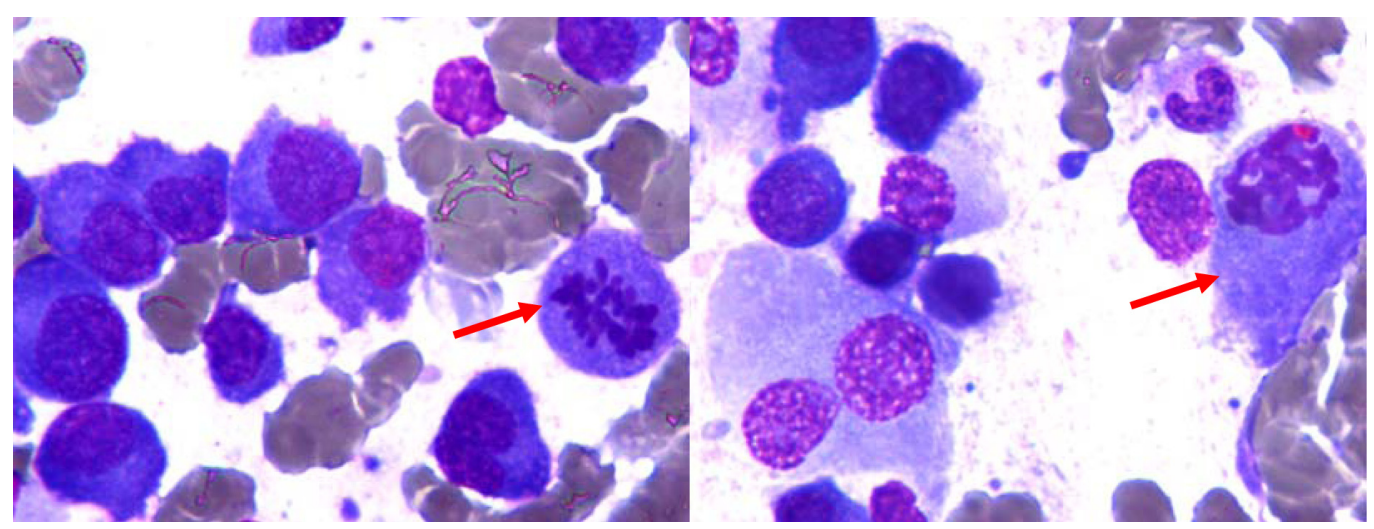

Figure 3 Bone marrow smears from a newly diagnosed multiple myeloma patient. Mitotic features (arrows) are seen among plasma cells. This is a rare phenomenon.

Fluorescence in situ hybridization (FISH) is more sensitive for recognizing specific chromosomal changes in quiescent cells and increases the proportion of detection of chromosomal abnormalities in MM up to more than $90 \%{ }^{42}$

Polymerase chain reaction and single nucleotide polymorphism detect the expression of given gene alterations.

Gene expression profiling (GEP) is the measurement of the activity (expression) of thousands of genes at once, to create a global picture of cellular function. When the given involved genes are recognized, DNA microarrays can monitor their levels of expression in parallel..$^{43}$ The microarrayGEP represent an excellent tool for studying MM because it does not only study the malignant plasma cell per se, but also its interactions with the BM microenvironment, a critical component of MM pathophysiology. ${ }^{44}$

\section{Primary events}

\section{Translocations involving the lgH locus and putative genes involved}

These translocations represent primary events and are considered by most investigators insufficient to give rise to full blown myeloma given that they are evenly distributed between MGUS, smoldering MM, and overt MM. ${ }^{41}$ By interface FISH analysis, IgH translocations are found in approximately $47 \%$ of MGUS patients, in $60 \%$ to $70 \%$ of patients with intramedullary MM, and in more than $80 \%$ of patients with plasma cell leukemia. ${ }^{45}$ The most frequent partners involving $14 \mathrm{q} 32$ are 11q13 (15\%), 4p16 (15\%), 16q23 (5\%), 20q11 (2\%), and 6p21 (5\%); two additional partners are also found rarely 12 p13 $(<1 \%)$ and 8q24 $(<1 \%) .{ }^{46}$ Thus seven recurrent oncogenes are involved in 14q32 translocations: CCND1, CCND2, CCND3, MAF, MAFB, MAFA, and FGFR3/MMSET. ${ }^{47}$ These are found in about $40 \%$ of myeloma patients, most of whose are nonhyperdiploid. ${ }^{48}$
Translocations involving the expression of cyclin D and consequently of cell cycle regulation seem to play an important role during primary transformation. It was initially found by FISH studies that the $\mathrm{t}(11 ; 14)$ is present in about $15 \%-20 \%$ of patients ${ }^{45,49}$ and is also common in MGUS and primary systemic amyloidosis. It results in upregulation of cyclin D1. It is the same translocation as in mantle cell lymphoma but IgH breakpoints are different (IgHS vs IgHJ). The $\mathrm{t}(11 ; 14)$ is peculiar in MM because, in contrast to other IgH translocations, it may involve the VDJ and switch regions suggesting that it might be a very early event, possibly an initiating one. Patients with $t(11 ; 14)$ are more likely to have a pseudodiploid karyotype; they present less frequent bone lesions and a better OS and response to treatment. ${ }^{50}$ The morphology of BM infiltrating cells is usually mature or lymphoplasmacytic. The $\mathrm{t}(6 ; 14)(\mathrm{p} 21 ; \mathrm{q} 32)$ translocation results in dysregulation of cyclin D3 and is detected in $3 \%-5 \%$ of MM cases exclusively by FISH. ${ }^{51}$ It leads to dysregulation of the c-maf proto oncogene and is usually associated with $13 q$ deletion. In spite of the fact that translocations $\mathrm{t}(11 ; 14)$ and $\mathrm{t}(6 ; 14)$ are encountered in about $30 \%$ by FISH, genomic profiling in a large cohort of patients with newly diagnosed disease revealed that almost all myeloma plasma cells exhibited increased and/or deregulated expression of either $C C N D 1, C C N D 2$, or $C C N D 3$, but this was not the case of plasma cells from the BM of healthy donors. ${ }^{40}$ Thus, cyclin D (D1, D2, or D3) deregulated expression is considered a generalized phenomenon linked to pathogenetic mechanisms and prognosis.

The other common $\operatorname{IgH}$ translocations is $\mathrm{t}(4 ; 14)$ (p16.3;q32), found in approximately in 15\% of MM patients using FISH. ${ }^{45}$ It is an unfavorable prognostic factor for patients treated with either conventional or high dose chemotherapy. Conventional karyotyping cannot detect this translocation because of its telomeric location. Tyrosine 
kinase receptor (FGFR3) and novel gene homologous to a Drosophila dysmorphy gene (MMSET) are in opposite transcriptional orientation at $4 \mathrm{p} 16 .^{52}$ Both are involved in $\mathrm{t}(4 ; 14)$. The translocation generates two fusion genes, IGH-MMSET on der(4) and FGFR3-IGH on der(14).

Other translocations involving the IgH locus have been observed at lower frequencies. $\mathrm{t}(14 ; 20)(\mathrm{q} 32 ; \mathrm{q} 11)$ is present in $2 \%$ of the patients with MM. The gene involved is MAFB that is a leukin zipper transcription factor. The $\mathrm{t}(9 ; 14)(\mathrm{p} 13 ; \mathrm{q} 32)$ translocation regulates the PAX-5 gene at $9 \mathrm{p} 13$ and has been found very rarely in MM patients in contrast to other plasma cell dyscrasias. ${ }^{20,53}$

\section{I $3 q$ deletion}

The role of $13 q$ deletion in the progression of $M M$ is still a matter of debate. A high incidence of $13 \mathrm{q}$ deletions has been reported in patients with MM with a preceding history of MGUS, suggesting that it may be involved in the transformation of MGUS into myeloma. ${ }^{17,27}$ Del13 was originally detected in approximatively $50 \%$ of patients with abnormal karyotypes, ${ }^{54}$ and has been associated with adverse prognosis. With the use of interphase FISH, it was shown that del13 may occur in all stages of the plasma cell neoplasms including MGUS and smoldering $\mathrm{MM},{ }^{27}$ the reported prevalence of monosomy or partial deletion of chromosome 13 in MM being of about 50\%. ${ }^{55}$ The aforementioned aberration involves the gene RB1. Resulting retinoblastoma protein (pRB) suppresses the $\mathrm{G} 1 \rightarrow \mathrm{S}$ transition in the cell cycle. It acts by inhibiting the E2F mediated transactivation of genes involved in DNA synthesis initiation (c-myc, b-myb, $\mathrm{cdc} 2$, dihydrofolate reductase, and thymidine kinase). $\mathrm{pRB}$ function depends on phosphorylation: hypophosphorylated $\mathrm{pRB}$ induces growth arrest while phosphorylated $\mathrm{pRB}$ cannot bind E2F. Myeloma cells exhibit strong expression of phosphorylated $\mathrm{pRB}$ and it was suggested that IL- 6 promotes MM cell proliferation by enhancing phosphorylation of $\mathrm{pRB} .{ }^{56}$ However, 13q14.3 deletions have been observed without RB1 loss, which means that RB1 is not the only critical locus of $13 q 14.3$ subband. ${ }^{57}$ It seems that the adverse prognostic effect of $13 \mathrm{q}$ deletions, as detected by FISH, is related to other associated abnormalities, such as $\mathrm{t}(4 ; 14)$ translocation and a partial deletion on chromosome $17 \mathrm{p} ;{ }^{42,57}$ consequently, patients who have only a chromosome 13 deletion have the same prognosis as those who do not have this abnormality.

\section{Secondary genetic events}

During the course of the disease, numerous events may take place contributing to the transformation of MM plasmacyte into a more aggressive one. Here, we will briefly present the three main disease progression aberrations.

\section{Chromosome I anomalies}

The short arm of chromosome 1 is involved in deletions, while the long arm in gains. Gains of the $\mathrm{q}$ arm of chromosome 1 are one of the most common genetic abnormalities in $\mathrm{MM}$ and are frequently associated with disease progression. 1q21 amplifications were linked to inferior survival, ${ }^{58}$ although it was not an independent factor in another study. ${ }^{59}$ It was suggested that the increased proliferation observed in the presence of $1 \mathrm{q} 21$ sequences is due to the increased expression of $C K S 1 B$ gene. ${ }^{60}$

\section{Secondary IgH translocations}

The $\mathrm{t}(8 ; 14)(\mathrm{q} 24 ; \mathrm{q} 32)$ translocation involves the proto oncogene c-myc which controls proliferation, differentiation, and apoptosis of neoplastic cells. C-myc rearrangements have been detected with FISH in about $15 \%$ of myeloma patients and $55 \%$ of myeloma cell lines. ${ }^{61}$ These abnormalities include unusual and complex translocations and insertions that often juxtapose myc with an $\operatorname{IgH}$ or $\operatorname{IgL}$ locus. ${ }^{62}$ It seems to be a late event during the progression of MM.

\section{p53 inactivation and chromosome 17 loss}

p53 inactivation by either deletion or mutation is a rare event in $\mathrm{MM}$ at diagnosis but the frequency increased during disease course. ${ }^{63}$ p53 is contained within the deleted region on $17 \mathrm{p} 13$ but questions remain regarding it being the critical gene. ${ }^{41}$ Deletions of 17 p13 are detectable in $8 \%-10 \%$ of patients and have been associated with stage III disease and significantly shorter survival. ${ }^{64}$

\section{Genetic findings related to treatment}

Effective treatment modalities are now available but their potency greatly varies among patients and genetic alterations have been related to theses discrepancies. Unsatisfactory response duration was observed after high dose treatment and BM transplantation or stem cell rescue in patients with partial or complete deletions of chromosome 13 by conventional cytogenetics, ${ }^{65} \mathrm{t}(4 ; 14)(\mathrm{p} 16 ; \mathrm{q} 32), \mathrm{t}(14 ; 16)(\mathrm{q} 32 ; \mathrm{q} 23)$ and $17 \mathrm{p} 13$ deletions, ${ }^{42,50}$ deletions and gains of chromosome $1 \mathrm{p} 21,{ }^{66}$ or amplification of its associated gene $C K S 1 B{ }^{67}$

Patients with the aforementioned genetic alterations also present a worse event-free and overall survival when treated with thalidomide; however the last is controversial and the drug can reverse the poor prognosis associated with cyclin-D1 
negativity and fibroblast growth factor-3 positivity. ${ }^{68}$ With regard to lenalidomide, that was relatively recently (early 2008) approved in Europe for the treatment of relapse/ refractory MM patients, fewer studies on its effectiveness in the presence of cytogenetic findings conferring adverse prognosis are available. A recent study on 100 newly diagnosed patients that received front-line lenalidomide showed that the 16 displaying poor prognosis cytogenetic features had a shorter progression-free survival. ${ }^{69}$

Bortezomib is a boronic acid dipeptide that reversibly and selectively inhibits the proteasome and degrades primarily ubiquitinated proteins. ${ }^{71}$ Many important target proteins of the proteasome, implicated in MM biology, have been identified, including cyclins A, B, D, and E, the tumor suppressor protein $\mathrm{p} 53$, pro-apoptotic protein Bax, ${ }^{72}$ cyclin-dependent kinase inhibitor $\mathrm{p} 27,{ }^{73}$ and the NF- $\mathrm{KB}$ inhibitor $\mathrm{I}-\kappa \mathrm{B} .{ }^{74}$ Indeed, studies have shown that bortezomib may overcome the adverse effect of deleterious genetic aberrations $13 \mathrm{q}$ deletions, $\mathrm{t}(4 ; 14)$, amplification $\mathrm{CKS} 1 \mathrm{~B},{ }^{74-76}$ but although outcomes are better than with other modalities, patients ultimately relapse.

Other drugs with a targeted mode of action will be released or approved soon (eg, mTOR inhibitors for the subset of patients with cyclin $\mathrm{D}_{1}$ dysregulation) and the therapeutic impact in MM is expected with interest.

\section{Additional genetic factors regulating $M M$ oncogenesis} Additional genes involved

Many other genes besides cyclin D,p53,c-myc, and CKS1B, as stated before, play a possible role in MM pathogeny and will not be further analyzed in the present context. The main are $N$ - and $K$-Ras mutations and genes involved in NFאB constitutive activation. $N$ - and $K$-Ras mutations are found in a considerable percentage in newly diagnosed patients (about 40\%). ${ }^{77}$ On the contrary they are rarely found in MGUS suggesting that they may contribute to or reflect a transforming process. ${ }^{78}$ The genetic background that produces constitutive activation of $\mathrm{NF} \kappa \mathrm{B}$, an important process in $\mathrm{MM}$, is a matter of ongoing research. ${ }^{79}$

\section{miRNAs}

Despite recent advances in oncogenomics and MM cellstroma interactions, further studies are needed to identify critical players in MM pathogenesis. It seems that small noncoding RNAs, previously thought to be unimportant, play an important role in the regulation of oncogenes that, in turn, participate to disease transformation. These, termed microRNAs (miRNAs) are noncoding RNAs of 19 to 25 nucleotides in length that regulate gene expression by inducing translational inhibition and degradation of their target mRNAs through base pairing to partially or fully complementary sites. ${ }^{80}$ miRNAs are involved in critical biological processes, including development, cell differentiation, stress response, apoptosis and proliferation. ${ }^{80}$ Recently, specific miRNA expression patterns have been linked to hematopoiesis and cancer. ${ }^{81}$ It was shown that in MM, miRNAs contribute to the malignant transformation of plasma cells. ${ }^{82}$ Upregulation of 60 and downregulation of 96 miRNAs was observed in CD138+ cells from MM patients and cell lines compared to healthy controls. Several of the aforementioned upregulated miRNAs targeted the p300-CBP-associated factor, a positive regulator of $\mathrm{p} 53$. In addition, miR-19a and miR-19b that specifically target SOCS-1, a negative regulator of the IL-6R/STAT-3 pathway, were upregulated by 100 -fold in patients' samples and by more than 2000-fold in MM cell lines while they were almost always absent in normal plasma cells and MGUS cells. The miRNAs may also disable disease progression. miR-15a and -16 were found to negatively regulate the proliferation and growth of MM cells in vitro and in vivo by inhibiting various kinase pathways. ${ }^{83}$ Moreover, miRNAs-15a and -16 exerted anti-MM activity even in the presence of BM microenviroment, thus representing potential therapeutical factors. As well as miRNAs may modulate gene expression, the opposite may also be true. The Argonaute- 2 gene, a regulator of miRNAs maturation and B-cell development, was recently identified as a uniformly over-expressed factor in all high risk MM plasma cell samples. ${ }^{84}$

\section{Prognostic genetic classifications}

Since Durie and Salmon classification, ${ }^{85}$ many other prognostic systems has been developed in an attempt to predict disease behavior and consequently to treat in the best way. Among them, numerous ones included the described above adverse genetic findings, ${ }^{38}$ namely hypodiploidy, deletion 13 by conventional karyotype, $\mathrm{t}(4 ; 14), \mathrm{t}(14 ; 16)$, and del17p13 by FISH. An international consensus genetic classification was recently published ${ }^{86}$ that provides recommendations for genetic testing in clinical practice.

The evolution of technology GEP-derived classifications have emerged, the two main of which are briefly described below.

Based on the knowledge that primary events are mainly the five recurrent IgH translocations, specific trisomies and the almost uniform expression of cyclin D genes, a classification 
scheme, based on the GEP spikes, was developed (referred to as TC classification). Reducing the complexity of the microarray from over 5000 probes to less than 30 genes, eight groups were identified: four with the primary translocations (4p16, 11q13, 6p21, Maf), three with CCND1 and CCND2 over-expression $\left(D_{1}, D_{1} \& D_{2}, D_{2}\right)$, and the last with the rare cases that do not over-express any cyclin D genes ('none'). Significant differences in global GEP profiles and clinical features were observed among the different groups. ${ }^{19}$

Complementing this supervised approach, unsupervised hierarchical clustering allows samples to self organize based on underlying correlations in gene expression patterns. Seven different and reproducible biologic-prognostic groups were recognized. ${ }^{89}$ These molecular groups are the MS, MF, CD-1, CD-2, HY, LB, and PR (referred to as UAMS classification). They present some similarities with the TC groups. Four of them (MF, MS, CD-1, and CD-2) are characterized by hyper-elevated expression that results from recurrent chromosomal translocations present in approximately $40 \%$ of MM, which occur as a result of errors in switch recombination and/or SHMs. The HY group is characterized by low level ectopic expression of CCND1 and over-expression of genes mapping - numbered to odd chromosomes that typically exhibit trisomy in MM. The LB (low-bone) group is characterized by a low incidence of magnetic resonance imaging-defined bone lesions and expresses high levels of CCND2 and a unique gene set, including endothelin-1.The last group, PR (proliferation) is not related to a primary genetic lesion but to a high expression level of proliferation genes and has the highest yield of metaphase cytogenetic abnormalities of all groups. Differences in clinical and laboratory findings as well as in survival were noted between the subgroups. The survival of the CD-1, CD-2, LB, and HY groups was better than the others.

Subsequently, many other models based on gene expression patterns have been developed, built with the purpose to better differentiate patients and to administer the most adequate treatment. However to date, there is still no model showing definitely clear cut results.

\section{Conclusion}

Progress has been made in the understanding of myeloma molecular pathogenesis. Recurrent cytogenetic abnormalities were found to contribute to the prognostication of disease behavior, survival and response to treatment. New drugs, such as bortezomib, were shown able to reverse the adverse impact of genetic alterations, with regard to response to treatment. However, patients almost always relapse, and the ultimate goal to use genetic findings to develop targeted therapies and cure MM, has not been reached yet.

Indeed, if it is true that MM precursor cell is a longlived B-cell memory cell, then modalities against it should be envisaged. Further studies are needed to clarify this possibility.

\section{Disclosure}

The authors report no conflicts of interest in this work.

\section{References}

1. Sahota S, Hamblin T, Oscier DG, Stevenson FK. Assessment of the role of clonogenic B lymphocytes in the pathogenesis of multiple myeloma. Leukemia. 1994;8(8):1285-1289.

2. Hallek M, Bergsagel PL, Anderson KC. Multiple myeloma: increasing evidence for a multistep transformation process. Blood. 1998;91(1):3-21.

3. Dalton WS, Bergsagel PL, Kuehl WM, Anderson KC, Harousseau JL. Multiple myeloma. Hematology Am Soc Hematol Educ Program. 2001:157-177.

4. Drewinko B, Alexanian R, Boyer H, Barlogie B, Rubinow SI. The growth fraction of human myeloma cells. Blood. 1981;57(2):333-338.

5. King MA, Nelson DS. Tumor cell heterogeneity in multiple myeloma: antigenic, morphologic, and functional studies of cells from blood and bone marrow. Blood. 1989;73(7):1925-1935.

6. Huff CA, Matsui W. Multiple myeloma cancer stem cells. J Clin Oncol. 2009;26(17):2895-2900.

7. Kristinsson SY, Goldin LR, Bjorkholm M, Koshiol J, Turesson I, Landgren $\mathrm{O}$. Genetic and immune-related factors in the pathogenesis of lymphoproliferative and plasma cell malignancies. Haematologica. 2009;94(11):1581-1589.

8. Vande Broek I, Vanderkerken K, van Camp B, van Riet I. Extravasation and homing mechanisms in multiple myeloma. Clin Exp Metastasis. 2008;25(4):325-334.

9. Yaccoby S, Pearse RN, Johnson CL, Barlogie B, Choi Y, Epstein J. Myeloma interacts with the bone marrow microenvironment to induce osteoclastogenesis and is dependent on osteoclast activity. $\mathrm{Br} J$ Haematol. 2002;116(2):278-290.

10. Mitsiades N, Mitsiades C, Munshi N, Richardson P, Anderson K. The role of the bone microenvironment in the pathophysiology and therapeutic management of multiple myeloma: interplay of growth factors, their receptors, and stromal interactions. Eur J Cancer. 2006;42(11) 1564-1573.

11. Wijdenes J, Dore JM, Clement C, Vermot-Desroches C. CD138. J Biol Regul Homeost Agents. 2002;16(2):152-155.

12. Vacca A, Ribatti D, Roncali L, et al. Bone marrow angiogenesis and progression in multiple myeloma. Br J Haematol. 1994;87(3): 503-508.

13. Anargyrou K, Terpos E, Vassilakopoulos TP, et al. Normalization of the serum angiopoietin-1 to angiopoietin-2 ratio reflects response in refractory/resistant multiple myeloma patients treated with bortezomib. Haematologica. 2008;93(3):451-454.

14. Blair JM, Zhou H, Seibel MJ, Dunstan CR. Mechanisms of disease: roles of OPG, RANKL, and RANK in the pathophysiology of skeletal metastasis. Nat Clin Pract Oncol. 2006;3(1):41-49.

15. Ria R, Todoerti K, Berardi S, et al. Gene expression profiling of bone marrow endothelial cells in patients with multiple myeloma. Clin Cancer Res. 2009;15(17):5369-5378.

16. Todoerti K, Lisignoli G, Storti P, et al. Distinct transcriptional profiles characterize bone microenvironment mesenchymal cells rather than osteoblasts in relationship with multiple myeloma bone disease. Exp Hematol. 2009;Dec 4. [Epub ahead of print]. 
17. Avet-Loiseau H, Facon T, Daviet A, et al. 14q32 translocations and monosomy 13 observed in monoclonal gammopathy of undetermined significance delineate a multistep process for the oncogenesis of multiple myeloma. Intergroupe Francophone du Myelome. Cancer Res. 1999;59(18):4546-4550.

18. Kuehl WM, Bergsagel PL. Multiple myeloma: evolving genetic events and host interactions. Nat Rev Cancer. 2002;2(3):175-187.

19. Bergsagel PL, Kuehl WM, Than F, Sawyer J, Barlogie B, Saughnessy Jr J. Cyclin D dysregulation: an early and unifying pathogenic event in multiple myeloma. Blood. 2005;106(1):296-303.

20. Pangalis GA, Tzenou T, Kalpadakis C, et al. Biologic and clinical overlap of IgM-secreting lymphomas; focus on Waldenstrom's macroglobulinemia. Haematologica. 2007;92(6 Suppl 2):74-77.

21. Kyle RA, Therneau TM, Rajkumar SV, et al. A long-term study of prognosis in monoclonal gammopathy of undetermined significance. N Engl J Med. 2002;346(8):564-569.

22. Kyle RA, Therneau TM, Rajkumar SV, et al. Prevalence of monoclonal gammopathy of undetermined significance. $N$ Engl J Med. 2006;354(13):1362-1369.

23. Landgren O, Kyle RA, Pfeiffer RM, et al. Monoclonal gammopathy of undetermined significance (MGUS) consistently precedes multiple myeloma: a prospective study. Blood. 2009;113(22):5412-5417.

24. Weiss BM, Abadie J, Verma P, Howard RS, Kuehl WM. A monoclonal gammopathy precedes multiple myeloma in most patients. Blood. 2009;113(22):5418-5422.

25. Rajkumar SV, Kyle RA, Therneau TM, et al. Serum free light chain ratio is an independent risk factor for progression in monoclonal gammopathy of undetermined significance. Blood. 2005;106(3):812-817.

26. Katzmann JA, Abraham RS, Dispenzieri A, Lust JA, Kyle RA. Diagnostic performance of quantitative kappa and lambda free light chain assays in clinical practice. Clin Chem. 2005;51(5):878-881.

27. Avet-Loiseau H, Li JY, Morineau N, et al. Monosomy 13 is associated with the transition of monoclonal gammopathy of undetermined significance to multiple myeloma. Blood. 1999;94(8):2583-2589.

28. Landgren O, Kyle RA. Multiple myeloma, chronic lymphocytic leukemia, and associated precursor diseases. Br J Haematol. 2009;139(5): 717-723.

29. Bataille R, Harouseau JL. Multiple myeloma. New Engl J Med. 1997;336(23):1657-1664.

30. Bradwell AR, Mead GP, Carr-Smith HD. 3rd ed. Serum Free Light Chain Analysis (Plus Hevylite). Birmingham, UK: The Binding Site Ltd; 2007:106-116.

31. Attal M, Huguet F, Schlaifer D, et al. Intensive combined therapy for previously untreated aggressive myeloma. Blood. 1992;79(5):1130-1136.

32. Barlogie B, Shaughnessy J, Tricot G, et al. Treatment of multiple myeloma. Blood. 2004;103(1):20-32.

33. Dimopoulos MA, Kastritis E. The role of novel drugs in multiple myeloma. Ann Oncol. 2008;19(Suppl 7):vii121-vii127.

34. Richardson P, Mitsiades C, Schlossman R, et al. The treatment of relapsed and refractory multiple myeloma. Hematology Am Soc Hematol Educ Program. 2007:317-323.

35. Kyrtsonis MC, Maltezas D, Koulieris E, et al. Response to bortezomib in refractory/relapsed multiple myeloma patients: a single center experience with discussion on specific issues. APJOH. 2010;1(4).

36. Fonseca R. Strategies for risk-adapted therapy in myeloma. Hematology Am Soc Hematol Educ Program. 2007:304-310.

37. Dispenzieri A, Rajkumar V, Gertz M, et al. Treatment of newly diagnosed multiple myeloma based on Mayo Stratification of Myeloma And Risk-adapted Therapy (mSMART): consensus statement. Mayo Clin Proc. 2007;82(3):323-341.

38. Kyrtsonis MC, Maltezas D, Tzenou T, Koulieris E, Bradwell AR. Staging systems and prognostic factors as a guide to therapeutic decisions in multiple myeloma. Semin Hematol. 2009;46(2):110-117.

39. Fonseca R, Barlogie B, Betaille R, et al. Genetics and cytogenetics of multiple myeloma: a workshop report. Cancer Res. 2004;64(4): $1546-1558$.
40. Bergsagel PL, Kuehl WM. Molecular pathogenesis and a consequent classification of multiple myeloma. J Clin Oncol. 2005;23(26): 6333-6338.

41. Chng WJ, Glebov O, Bergsagel PL, Kuehl WM. Genetic events in the pathogenesis of multiple myeloma. Best Pract Res Clin Haematol. 2007;20(4):571-596.

42. Avet-Loiseau H, Attal M, Moreau P, et al. Genetic abnormalities and survival in multiple myeloma: the experience of the Intergroupe Francophone du Myelome. Blood. 2007;109(8):3489-3495.

43. Schena M, Shalon D, Davis RW, Brown PO. Quantitative monitoring of gene expression patterns with a complementary DNA microarray. Science. 1995;270(5235):467-470.

44. Dalton WS. The tumor microenviroment: focus on myeloma. Cancer Treat Rev. 2003;29(Suppl 1):11-19.

45. Avet-Loiseau H, Li JY, Facon T, et al. High incidence of translocations $\mathrm{t}(11 ; 14)(\mathrm{q} 13 ; \mathrm{q} 32)$ and $\mathrm{t}(4 ; 14)(\mathrm{p} 16 ; \mathrm{q} 32)$ in patients with plasma cell malignancies. Cancer Res. 1998;58(24):5640-5645.

46. Hanamura I, Iida S, Ueda R, et al. Identification of three novel chromosomal translocation partners involving the immunoglobulin loci in newly diagnosed myeloma and human myeloma cell lines. Blood (ASH Annual Meeting Abstracts). 2005;106, Abstract 1552.

47. Gabrea A, Bersagel PL, Kuehl WM. Distinguishing primary and secondary translocations in multiple myeloma. DNA Repair. 2006;5: 1225-1233.

48. Zhan F, Huang Y, Collins S, et al. The molecular classification of multiple myeloma. Blood. 2006;108(6):2020-2028.

49. Fonseca R, Witzig TE, Gertz MA, et al. Multiple myeloma and the translocation $\mathrm{t}(11 ; 14)(\mathrm{q} 13 ; \mathrm{q} 32)$ : a report on 13 cases. Br J Haematol. 1998;101(2):296-301.

50. Gertz M, Lacy M, Dispenzieri A, et al. Clinical implications of $\mathrm{t}(11 ; 14)$ $(\mathrm{q} 13 ; \mathrm{q} 32), \mathrm{t}(4 ; 14)(\mathrm{p} 16.3 ; \mathrm{q} 32)$, and $-17 \mathrm{p} 13$ in myeloma patients treated with high-dose therapy. Blood. 2005;106(8):2837-2840.

51. Shaughnessy Jr J, Gabrea A, Qi Y, et al. Cyclin D3 at 6p21 is dysregulated by recurrent chromosomal translocations to immunoglobulin loci in multiple myeloma. Blood. 2001;98(1):217-223.

52. Lauring J, Abukhdeir A, Konishi H, et al. The multiple myeloma associated MMSET gene contributes to cellular adhesion, clonogenic growth, and tumorigenicity. Blood. 2008;111(2):856-864.

53. Lida S, Rao PH, Nallasivam $P$, et al. The $t(9 ; 14)(\mathrm{p} 13 ; \mathrm{q} 32)$ chromosomal translocation associated with lymphoplasmacytoid lymphoma involves the PAX-5 gene. Blood. 1996;88(11):4110-4117.

54. Sawyer JR, Waldron JA, Jagannath S, Barlogie B. Cytogenetic findings in 200 patients with multiple myeloma. Cancer Genet Cytogenet. 1995;82(1):41-49.

55. Fonseca R, Harrington D, Oken MM, et al. Biological and prognostic significance of interphase fluorescence in situ hybridization detection of chromosome 13 abnormalities $(\Delta 13)$ in multiple myeloma. An Eastern Cooperative Oncology Group study. Cancer Res. 2002;62(3):715-720.

56. Urashima M, Ogata A, Chauhan D, et al. Interleukin-6 promates multiple myeloma cell growth via phosphorylation of retinoblastoma protein. Blood. 1996;88(6):2219-2227.

57. Shaughnessy Jr J, Zhan F, Burrington BE, et al. A validated gene expression model of high-risk multiple myeloma is defined by deregulated expression of genes mapping to chromosome 1. Blood. 2007;109(6): 2276-2284.

58. Hanamura I, Stewart JP, Huang Y, et al. Frequent gain of chromosome band 1q21 in plasma-cell dyscrasias detected by fluorescence in situ hybridization: incidence increases from MGUS to relapsed myeloma and is related to prognosis and disease progression following tandem stem-cell transplantation. Blood. 2006;108(6):1724-1732.

59. Fonseca R, van Wier SA, Chng WJ, et al. Prognostic value of chromosome 1q21 gain by fluorescent in situ hybridization and increase CKS1B expression in myeloma. Leukemia. 2006;20(11):2034-2040.

60. Zhan F, Colla S, Wu X, et al. CKS1B, over expressed in aggressive disease, regulates multiple myeloma growth and survival through SKP2- and p27Kip1-dependent and independent mechanisms. Blood. 2007;109(11):4995-5001. 
61. Avet-Loiseau H, Gerson F, Magrangeas F, Minvielle S, Harousseau JL, Bataille R. Rearrangements of the c-myc oncogene are present in $15 \%$ of primary human multiple myeloma tumors. Blood. 2001;98(10): 3082-3086.

62. Shou Y, Martelli ML, Gabrea A, et al. Diverse karyotypic abnormalities of the c-myc locus associated with c-myc dysregulation and tumor progression in multiple myeloma. Proc Natl Acad Sci USA. 2000;97(1): 228-233.

63. Chng WJ, Price-Troska T, Gonzalez-Paz N, et al. Clinical significance of TP53 mutation in myeloma. Leukemia. 2007;21(3):582-584.

64. Neri A, Baldini L, Trecca D, Cro L, Polli E, Maiolo AT. p53 gene mutations in multiple myeloma are associated with advanced forms of malignancy. Blood. 1993;81(1):128-135.

65. Tricot G, Barlogie B, Jagannath S, et al. Poor prognosis in multiple myeloma is associated only with partial or complete deletions of chromosome 13 or abnormalities involving 11q and not with other karyotype abnormalities. Blood. 1995;86(11):4250-4256.

66. Chang H, Qi X, Jiang A, Xu W, Young T, Reece D. 1p21 deletions are strongly associated with 1q21 gains and are an independent adverse prognostic factor for the outcome of high-dose chemotherapy in patients with multiple myeloma. Bone Marrow Transplant. 2010;45(1):117-121.

67. Chang H, Qi X, Trieu Y, et al. Multiple myeloma patients with CKS1B gene amplification have a shorter progression-free survival post-autologous stem cell transplantation. Br J Haematol. 2006;135(4):486-491.

68. Kelly TW, Baz R, Hussein M, Karafa M, Cook JR. Clinical significance of cyclin D1, fibroblast growth factor receptor-3 and p53 immunohistochemistry in plasma cell myeloma treated with a thalidomide-based regimen. Hum Pathol. 2009;40(3):405-412.

69. Kapoor P, Kumar S, Fonseca R, et al. Impact of risk stratification on outcome among patients with multiple myeloma receiving initial therapy with lenalidomide and dexamethasone. Blood. 2009;114(3):518-521.

70. Pangalis GA, Kyrtsonis MC, Vassilakopoulos TP, et al. Immunotherapeutic and immunoregulatory drugs in haematologic malignancies. Curr Top Med Chem. 2006;6(16):1657-1686.

71. Li B, Dou QP. Bax degradation by the ubiquitin/proteasome dependent pathway: involvement in tumor survival and progression. Proc Natl Acad Sci US A. 2000;97(8):3850-3855.

72. Pagano M, Tam SW, Theodoras AM, et al. Role of the ubiquitinproteasome pathway in regulating abundance of the cyclin-dependent kinase inhibitor p27. Science. 1995;269(5224):682-685.

73. Hideshima T, Chauhan D, Richardson P, et al. NF-kappa B as a therapeutic target in multiple myeloma. J Biol Chem. 2002;277(19): 16639-16647.
74. Chang H, Trieu Y, Qi X, Xu W, Stewart KA, Reece D. Bortezomib therapy response is independent of cytogenetic abnormalities in relapsed/ refractory multiple myeloma. Leuk Res. 2007;31(6):779-782.

75. Jagannath S, Richardson PG, Sonneveld P, et al. Bortezomib appears to overcome the poor prognosis conferred by chromosome 13 deletion in phase 2 and 3 trials. Leukemia. 2007;21(1):151-157.

76. Sagaster V, Ludwig H, Kaufmann H, et al. Bortezomib in relapsed multiple myeloma: response rates and duration of response are independent of a chromosome 13q-deletion. Leukemia. 2007;21(1):164-168.

77. Bezieau S, Devilder MC, Avet-Loiseau H, et al. High incidence of $\mathrm{N}$ and $\mathrm{K}-$ Ras activating mutations in multiple myeloma and primary plasma cell leukemia at diagnosis. Hum Mutat. 2001;18(3):212-224.

78. Rasmussen T, Kuehl M, Lodahl M, Johnsen HE, Dahl IM. Possible roles for activating RAS mutations in the MGUS to MM transition and in the intramedullary to extramedullary transition in some plasma cell tumors. Blood. 2005;105(1):317-323.

79. Keats JJ, Fonseca R, Chesi M, et al. Promiscuous mutations frequently activate the noncanonical NF-KB pathway in multiple myeloma. Cancer Cell. 2007;12(2):131-144.

80. Bartel D. MicroRNAs: genomics, biogenesis, mechanism, and function. Cell. 2004;116(2):281-297.

81. Croce CM. Oncogenes and cancer. $N$ Engl J Med. 2008;358(5): $502-511$.

82. Pichiori F, Suh SS, Ladetto M, et al. MicroRNAs regulate critical genes associated with multiple myeloma pathogenesis. Proc Natl Acad Sci US A. 2008;105(35):12885-12890.

83. Roccaro A, Sacco A, Thompson B, Leleu X, et al. MicroRNAs 15a and 16 regulate tumor proliferation in multiple myeloma. Blood. 2009; 113(26):6669-6680

84. Zhou Y, Chen L, Barlogie B, et al. High risk myeloma is characterized by uniform over-expression of miRNAs and increased copy number and expression of Argonaute 2, a master regulator of miRNA maturation and B-cell development. Blood (ASH Annual Meeting Abstracts). 2009; $114: 1804$.

85. Durie BG, Salmon SE. A clinical staging system for multiple myeloma. Correlation of measured myeloma cell mass with presenting clinical features, response to treatment, and survival. Cancer. 1975;36(3): $842-854$.

86. Fonseca R, Bergsagel PL, Drach J, et al. International Myeloma Working Group molecular classification of multiple myeloma: spotlight review. Leukemia. 2009;23(12):2210-2221.
The Application of Clinical Genetics

\section{Publish your work in this journal}

The Application of Clinical Genetics is an international, peer-reviewed open access journal that welcomes laboratory and clinical findings in the field of human genetics. Specific topics include: Population genetics; Functional genetics; Natural history of genetic disease; Management of genetic disease; Mechanisms of genetic disease; Counselling and

\section{Dovepress}

ethical issues; Animal models; Pharmacogenetics; Prenatal diagnosis; Dysmorphology. The manuscript management system is completely online and includes a very quick and fair peer-review system, which is all easy to use. Visit http://www.dovepress.com/testimonials.php to read real quotes from published authors. 